Brief paper

\title{
Adaptive robot navigation with collision avoidance subject to 2nd-order uncertain dynamics
}

\author{
Christos K. Verginis*, Dimos V. Dimarogonas \\ School of Electrical Engineering and Computer Science, KTH Royal Institute of Technology, SE-100 44, Stockholm, Sweden
}

\section{A R T I C L E I N F O}

\section{Article history:}

Received 5 November 2019

Received in revised form 6 June 2020

Accepted 2 September 2020

Available online $\mathrm{xxxx}$

\section{Keywords:}

Robot navigation

Obstacle avoidance

Uncertain dynamics

Adaptive control

\begin{abstract}
A B S T R A C T
This paper considers the problem of robot motion planning in a workspace with obstacles for systems with uncertain 2nd-order dynamics. In particular, we combine closed form potential-based feedback controllers with adaptive control techniques to guarantee the collision-free robot navigation to a predefined goal while compensating for the dynamic model uncertainties. We base our findings on sphere world-based configuration spaces, but extend our results to arbitrary star-shaped environments by using previous results on configuration space transformations. Moreover, we propose an algorithm for extending the control scheme to decentralized multi-robot systems. Finally, extensive simulation results verify the theoretical findings.
\end{abstract}

(c) 2020 Elsevier Ltd. All rights reserved.

\section{Introduction}

Motion planning and specifically robotic navigation in obstacle-cluttered environments is a fundamental problem in the field of robotics (Lumelsky, 2005). Several techniques have been developed in the related literature, such as discretization of the continuous space and employment of discrete algorithms (e.g., Dijkstra, $\left.A^{\star}\right)$, probabilistic roadmaps, sampling-based motion planning, and feedback-based motion planning (LaValle, 2006). The latter, which is the focus of the current paper, offers closedform analytic solutions by usually evaluating appropriately designed artificial potential fields, avoiding thus the potential complexity of workspace discretization and the respective algorithms. At the same time, feedback-based methods provide a solution to the control aspect of the motion planning problem, i.e., the correctness based on the solution of the closed-loop differential equation that describes the robot model.

Early works on feedback-based motion planning established the Koditschek-Rimon navigation function (KRNF) (Koditschek \& Rimon, 1990; Rimon \& Koditschek, 1992), where, through gain

\footnotetext{
This work was supported by the H2020 ERC Starting Grant BUCOPHSYS, the European Union's Horizon 2020 Research and Innovation Programme under the GA No. 731869 (Co4Robots), the Swedish Research Council (VR), the Knut och Alice Wallenberg Foundation (KAW) and the Swedish Foundation for Strategic Research (SSF). The material in this paper was not presented at any conference. This paper was recommended for publication in revised form by Associate Editor C.C. Cheah under the direction of Editor Thomas Parisini.

* Corresponding author.

E-mail addresses: cverginis@kth.se (C.K. Verginis), dimos@kth.se (D.V. Dimarogonas).
}

tuning, the robot converges safely to its goal from almost all initial conditions (in the sense of a measure-zero set). KRNFs were extended to more general workspaces and adaptive gain controllers (Filippidis \& Kyriakopoulos, 2011), to multi-robot systems (Dimarogonas, Loizou, Kyriakopoulos, \& Zavlanos, 2006; Roussos \& Kyriakopoulos, 2013; Verginis \& Dimarogonas, 2017; Verginis, Xu, \& Dimarogonas, 2017), and more recently, to convex potential and obstacles (Paternain, Koditschek, \& Ribeiro, 2017). The idea of gain tuning has been also employed to an alternative KRNF in Tanner and Kumar (2005). Tuning-free constructions of artificial potential fields have also been developed in the related literature; Panagou (2017) tackles nonholonomic multi-robot systems, and in Loizou (2017) and Vlantis, Vrohidis, Bechlioulis, and Kyriakopoulos (2018) harmonic functions, also used in Szulczyński, Pazderski, and Kozłowski (2011), are combined with adaptive controllers to achieve almost global safe navigation. A transformation of arbitrarily shaped worlds to points worlds, which facilitates the motion planning problem, is also considered in Loizou (2017) and Vlantis et al. (2018) and in Loizou (2014) for multi-robot systems. The recent works Loizou (2017), Vrohidis, Vlantis, Bechlioulis, and Kyriakopoulos (2018) guarantee also safe navigation in predefined time.

Barrier functions for multi-robot collision avoidance are employed in Wang, Ames, and Egerstedt (2017) and optimizationbased techniques via model predictive control (MPC) can be found in Filotheou, Nikou, and Dimarogonas (2018), Mendes Filho, Lucet, and Filliat (2017), Morgan, Subramanian, Chung, and Hadaegh (2016) and Verginis, Nikou, and Dimarogonas (2018); AlonsoMora, Breitenmoser, Rufli, Siegwart, and Beardsley (2012) and Van Den Berg, Snape, Guy, and Manocha (2011) propose reciprocal collision obstacle by local decision making for the desired velocity of the robot(s). Ellipsoidal obstacles are tackled 
in Grushkovskaya and Zuyev (2018) and Stavridis, Papageorgiou, and Doulgeri (2017) extends a given potential field to 2nd-order systems. A similar idea is used in Montenbruck, Bürger, and Allgöwer (2015), where the effects of an unknown drift term in the dynamics are examined. Workspace decomposition methodologies with hybrid controllers are employed in Arslan, Guralnik, and Koditschek (2016), Arslan and Koditschek (2016), and Berkane, Bisoffi, and Dimarogonas (2019), and Huber, Billard, and Slotine (2019) employ a contraction-based methodology that can also tackle the case of moving obstacles.

A common assumption that most of the aforementioned works consider is the simplified robot dynamics, i.e., single integrators/unicycle kinematics, without taking into account any robot dynamic parameters. Hence, the actual robot trajectory might deviate from the desired one, jeopardizing its safety. Secondorder realistic robot models are considered in MPC-schemes, like Filotheou et al. (2018), Mendes Filho et al. (2017) and Verginis et al. (2018), which might, however, result in computationally expensive solutions. Moreover, regarding model uncertainties, a global upper bound is required, which is used to enlarge the obstacle boundaries and might yield infeasible solutions. A 2ndorder model is considered in Grushkovskaya and Zuyev (2018) and Stavridis et al. (2017), without, however, considering any unknown dynamic terms. The works Arslan and Koditschek (2017), Dimarogonas et al. (2006), Koditschek (1991) and Loizou (2011) consider simplified 2nd-order systems with known dynamic terms (and in particular, inertia and gravitational terms that are assumed to be successfully compensated); Montenbruck et al. (2015) guarantee the asymptotic stability of 2nd-order systems with a class of unknown drift terms to the critical points of a given potential function. However, there is no characterization of the region of attraction of the goal. Adaptive control for constant unknown parameters is employed in Cheah, Hou, and Slotine (2009), where a swarm of robots is controlled to move inside a desired region.

In this paper, we consider the robot navigation in an obstaclecluttered environment under 2nd-order uncertain robot dynamics, in terms of unknown mass and friction/drag terms. Our main contribution lies in the design of a novel 2nd-order smooth navigation function as well as an adaptive control law that guarantees the safe navigation of the robot from almost all initial conditions. We also show how the proposed scheme can be applied to starworlds, i.e., workspaces with star-shaped obstacles (Rimon \& Koditschek, 1992), as well as to decentralized multi-robot navigation. Adaptive control for multi-robot coordination was also employed in our previous works (Verginis \& Dimarogonas, 2017, 2019). The results in Verginis and Dimarogonas (2017), however, are only existential, since we do not provide an explicit potential function that satisfies the desired properties, while Verginis and Dimarogonas (2019) focus on the multi-agent ellipsoidal collision avoidance, without guaranteeing achievement of the primary task.

The rest of the paper is organized as follows: Section 2 provides the notation used throughout the paper. Section 3 describes the tackled problem and Section 4 provides the main results. Sections 5 and 6 extend the proposed scheme to star worlds and multi-agent frameworks, respectively. Finally, simulation studies are given in Section 7 and Section 8 concludes the paper.

\section{Notation}

The set of natural and real numbers is denoted by $\mathbb{N}$, and $\mathbb{R}$, respectively, and $\mathbb{R}_{\geq 0}^{n}, \mathbb{R}_{>0}^{n}, n \in \mathbb{N}$, are the $n$-dimensional sets of nonnegative and positive real numbers, respectively. The notation $\|x\|$ implies the Euclidean norm of a vector $x \in \mathbb{R}^{n}$. The identity matrix is denoted by $I_{n} \in \mathbb{R}^{n \times n}$, the $n \times m$ matrix of zeros by $0_{n \times m}$ and the $n$-dimensional zero vector by $0_{n}$. The gradient and Hessian of a function $f: \mathbb{R}^{n} \rightarrow \mathbb{R}$ are denoted by $\nabla_{x} f(x):=\frac{\partial f(x)}{\partial x} \in$ $\mathbb{R}^{n}$ and $\nabla_{x}^{2} f(x) \in \mathbb{R}^{n \times n}$, respectively.

\section{Problem statement}

Consider a spherical robot operating in a bounded workspace $\mathcal{W}$, characterized by its position vector $x \in \mathbb{R}^{n}, n \in\{2,3\}$ and radius $r>0$, and subject to the dynamics:

$\dot{x}=v$

$m \dot{v}+f(x, v)+m g=u$,

where $m>0$ is the unknown mass, $g \in \mathbb{R}^{n}$ is the constant gravity vector, $u \in \mathbb{R}^{n}$ is the input vector, and $f: \mathbb{R}^{2 n} \rightarrow \mathbb{R}^{n}$ is an $u n$ known friction-like function, satisfying the following assumption:

Assumption 1. The function $f: \mathbb{R}^{2 n} \rightarrow \mathbb{R}^{n}$ is analytic and satisfies

$\|f(x, v)\| \leq \alpha\|v\|$,

$\forall x, v \in \mathbb{R}^{2 n}$, where $\alpha \in \mathbb{R}_{\geq 0}$ is an unknown constant.

The aforementioned assumption is inspired by standard friction-like terms, which can be approximated by continuously differentiable velocity functions (Makkar, Dixon, Sawyer, \& Hu, 2005). Constant unknown friction terms could be also included in the dynamics (e.g., incorporated in the constant gravity vector). Note also that $\|f(x, v)\| \leq \alpha\|v\|$ implies $f\left(x, 0_{n}\right)=0_{n}$, and $\left.\frac{\partial f(x, v)}{\partial x}\right|_{v=0_{n}}=0_{n \times n}$. The workspace is assumed to be an open ball centered at the origin

$\mathcal{W}:=\left\{q \in \mathbb{R}^{n}:\|q\|<r_{\mathcal{W}}\right\}$,

where $r_{\mathcal{W}}>0$ is the workspace radius. The workspace contains $M \in \mathbb{N}$ closed sets $\mathcal{O}_{j}, j \in \mathcal{J}:=\{1, \ldots, M\}$, corresponding to obstacles. Each obstacle is a closed ball centered at $c_{j} \in \mathbb{R}^{3}$, with radius $r_{o_{j}}>0$, i.e., $\mathcal{O}_{j}:=\left\{q \in \mathcal{W}:\left\|q-c_{j}\right\| \leq r_{o_{j}}\right\}, \quad \forall j \in \mathcal{J}$. The analysis that follows will be based on the transformed workspace:

$\overline{\mathcal{W}}:=\left\{q \in \mathbb{R}^{n}:\|q\|<\bar{r}_{\mathcal{W}}:=r_{\mathcal{W}}-r\right\}$,

and set of obstacles $\overline{\mathcal{O}}_{j}:=\left\{q \in \mathcal{W}:\left\|q-c_{j}\right\| \leq \bar{r}_{o_{j}}:=r_{o_{j}}+r\right\}, \quad \forall j \in$ $\mathcal{J}$, where the robot is reduced to the point $x$. The free space is defined as

$\mathcal{F}:=\overline{\mathcal{W}} \backslash \bigcup_{j \in \mathcal{J}} \overline{\mathcal{O}}_{j}$,

also known as a sphere world (Koditschek \& Rimon, 1990). We consider the following common feasibility assumption (Koditschek \& Rimon, 1990; Vrohidis et al., 2018) for $\mathcal{F}$ :

Assumption 2. The workspace $\mathcal{W}$ and the obstacles $\mathcal{O}_{j}$ satisfy $\left\|c_{i}-c_{j}\right\|>r_{o_{i}}+r_{o_{j}}+2 r$ and $r_{\mathcal{W}}-\left\|c_{j}\right\|>r_{o_{j}}+2 r, \forall i, j \in \mathcal{J}, i \neq j$.

Assumption 2 implies that we can find some $\bar{r}>0$ such that

$\left\|c_{i}-c_{j}\right\|>r_{o_{i}}+r_{o_{j}}+2 r+2 \bar{r}, \quad \forall i, j \in \mathcal{J}, i \neq j$,

$r_{\mathcal{W}}-\left\|c_{j}\right\|>r_{o_{j}}+2 r+2 \bar{r}, \quad \forall j \in \mathcal{J}$

This paper treats the problem of navigating the robot to a destination $x_{\mathrm{d}}$ while avoiding the obstacles and the workspace boundary, formally stated as follows:

Problem 1. Consider a robot subject to the uncertain dynamics (1), operating in the aforementioned sphere world, with $\left(x\left(t_{0}\right)\right.$, $\left.v\left(t_{0}\right)\right) \in \mathcal{F} \times \mathbb{R}^{n}$. Given a destination $x_{\mathrm{d}} \in \mathcal{F}$, design a control protocol $u$ such that $x(t) \in \mathcal{F}, \quad t \geq t_{0}$ and $\lim _{t \rightarrow \infty}(x(t), v(t))=$ $\left(x_{\mathrm{d}}, 0_{n}\right)$. 


\section{Main results}

We provide in this section our methodology for solving Problem 1. Define first the set $\overline{\mathcal{J}}:=\{0\} \cup \mathcal{J}$ as well as the distances $d_{j}: \mathcal{F} \rightarrow \mathbb{R}_{\geq 0}, j \in \overline{\mathcal{J}}$, with $d_{j}(x):=\left\|x-c_{j}\right\|^{2}-\bar{r}_{o_{j}}^{2}, \forall j \in \mathcal{J}$, and $d_{0}(x):=\bar{r}_{\mathcal{W}}^{2}-\|x\|^{2}$. Note that, by keeping $d_{j}(x)>0, d_{0}(x)>0$, we guarantee that $x \in \mathcal{F}$. ${ }^{1}$ We also define the constant

$\bar{r}_{\mathrm{d}}:=\min \left\{\bar{r}_{\mathcal{W}}^{2}-\left\|x_{\mathrm{d}}\right\|^{2}, \min _{j \in \mathcal{J}}\left\{d_{j}\left(x_{\mathrm{d}}\right)\right\}\right\}$

as the minimum distance of the goal to the obstacles/workspace boundary. We introduce next the notion of the 2nd-order navigation function:

Definition 1. A 2nd-order navigation function is a function $\phi$ : $\mathcal{F} \rightarrow \mathbb{R}_{\geq 0}$ of the form

$\phi(x):=k_{1}\left\|x-x_{\mathrm{d}}\right\|^{2}+k_{2} \sum_{j \in \overline{\mathcal{J}}} \beta\left(d_{j}(x)\right)$,

where $\beta: \mathbb{R}_{>0} \rightarrow \mathbb{R}_{\geq 0}$ is a (at least) twice contin. differentiable function and $k_{1}, k_{2}$ are positive constants, with the followings properties:

(1) $\beta((0, \tau])$ is strictly decreasing, $\lim _{z \rightarrow 0} \beta(z)=\infty$, and $\beta(z)$ $=\beta(\tau), \forall z \geq \tau, j \in \overline{\mathcal{J}}$, for some $\tau>0$,

(2) $\phi(x)$ has a global minimum at $x=x_{\mathrm{d}} \in \operatorname{int}(\mathcal{F})$ where $\phi\left(x_{\mathrm{d}}\right)=0$,

(3) if $\beta^{\prime}\left(d_{k}(x)\right) \neq 0$ and $\beta^{\prime \prime}\left(d_{k}(x)\right) \neq 0$ for some $k \in \overline{\mathcal{J}}$, then $\beta^{\prime}\left(d_{j}(x)\right)=\beta^{\prime \prime}\left(d_{j}(x)\right)=0$, for all $j \in \overline{\mathcal{J}} \backslash\{k\}$.

(4) The function $\widetilde{\beta}:(0, \tau) \rightarrow \mathbb{R}_{\geq 0}$, with $\widetilde{\beta}(z):=\beta^{\prime \prime}(z) z \sqrt{z}$ is strictly decreasing.

By using the first property we will guarantee that, by keeping $\beta\left(d_{j}(x)\right)$ bounded, there are no collisions with the obstacles or the free space boundary. Property 2 will be used for the asymptotic stability of the desired point $x=x_{\mathrm{d}}$. Property 3 places the rest of the critical points of $\phi$ (which are proven to be saddle points) close to the obstacles, and the last property is used to guarantee that these are non-degenerate. An example for $\beta$ that satisfies properties (1) and (4), is

$\beta(z):= \begin{cases}\left(6 z^{5}-15 z^{4}+10 z^{3}\right)^{-1}, & z \leq 1 \\ 1, & z \geq 1,\end{cases}$

Note that $\beta$ is essentially a reciprocal barrier function (Wang et al., 2017). We prove next that, by appropriately choosing $\tau$, only one $\beta\left(d_{j}(x)\right), j \in \overline{\mathcal{J}}$ affects the robotic agent for each $x \in$ $\mathcal{F}$, and furthermore that $\beta^{\prime}\left(d_{j}\left(x_{\mathrm{d}}\right)\right)=\beta^{\prime \prime}\left(d_{j}\left(x_{\mathrm{d}}\right)\right)=0$. Hence, properties (2) and (3) of Definition 1 are satisfied.

Proposition 1. By choosing $\tau$ as $\tau \in\left(0, \min \left\{\bar{r}^{2}, \bar{r}_{\mathrm{d}}\right\}\right)$, where $\bar{r}, \bar{r}_{\mathrm{d}}$ were introduced in (6) and (7), respectively, we guarantee that at each $x \in \mathcal{F}$ there is no more than one $j \in \overline{\mathcal{J}}$ such that $d_{j} \leq \tau$, implying that $\beta^{\prime}\left(d_{j}(x)\right)$ and $\beta^{\prime \prime}\left(d_{j}(x)\right)$ are non-zero.

Proof. See the proof of Prop. 1 in Verginis and Dimos (2020).

Moreover, one can easily conclude in view of (7) that $\beta^{\prime}\left(d_{j}\left(x_{\mathrm{d}}\right)\right)$ $=\beta^{\prime \prime}\left(d_{j}\left(x_{\mathrm{d}}\right)\right)=0, \forall j \in \overline{\mathcal{J}}$.

Intuitively, the obstacles and the workspace boundary have a local region of influence defined by the constant $\tau$, which will play a significant role in determining the stability of the overall scheme later. This robot interaction with only one obstacle at a time has also been demonstrated in the feedback control-based

\footnotetext{
1 A safety margin can also be included, which needs, however, to be incorporated in the constant $\bar{r}$ of (6).
}

related literature, e.g., Arslan and Koditschek (2016), Filippidis and Kyriakopoulos (2011), Lionis, Papageorgiou, and Kyriakopoulos (2007), Paternain et al. (2017) and Vrohidis et al. (2018), which deals with simplified single-integrator models, as well as in the more discrete decision making bug algorithms (Lumelsky, 2005), which involve circumnavigation of obstacles and can handle in general complex unknown environments.

Given the aforementioned definitions, we design a reference signal $v_{\mathrm{d}}: \mathcal{F} \rightarrow \mathbb{R}^{n}$ for the robot velocity $v$ as

$v_{\mathrm{d}}(x)=-\nabla_{x} \phi(x)$.

Next, we design the control input $u$ to guarantee tracking of the aforementioned reference velocity as well as compensation of the unknown terms $m$ and $f(x, v)$. More specifically, we define the signals $\hat{m} \in \mathbb{R}$ and $\hat{\alpha} \in \mathbb{R}$ as the estimation terms of $m$ and $\alpha$ (see Assumption 1), respectively, and the respective errors $\tilde{m}:=\hat{m}-m, \widetilde{\alpha}:=\hat{\alpha}-\alpha$. We design now the control law $u: \mathcal{F} \times \mathbb{R}^{n+2} \rightarrow \mathbb{R}^{n}$ as $u:=u(x, v, \hat{m}, \hat{\alpha})$, with

$u:=-k_{\phi} \nabla_{x} \phi(x)+\hat{m}\left(\dot{v}_{\mathrm{d}}+g\right)-\left(k_{v}+\frac{3}{2} \hat{\alpha}\right) e_{v}$,

where $e_{v}:=v-v_{\mathrm{d}}$, and $k_{v}, k_{\phi}$ are positive gain constants. Moreover, we design the adaptation laws for the estimation signals as

$\dot{\hat{m}}:=-k_{m} e_{v}^{\top}\left(\dot{v}_{\mathrm{d}}+g\right)$
$\dot{\hat{\alpha}}:=k_{\alpha}\left\|e_{v}\right\|^{2}$,

with $k_{m}, k_{\alpha}$ positive gain constants, $\hat{\alpha}\left(t_{0}\right) \geq 0$, and arbitrary finite initial condition $\hat{m}\left(t_{0}\right)$. The choices for the control and adaptation laws are based on Lyapunov techniques, and follow standard adaptive control methodologies (see, e.g., Lavretsky \& Wise, 2013). The following theorem establishes the correctness of the proposed control protocol.

Theorem 1. Consider a robot operating in $\mathcal{W}$, subject to the uncertain 2nd-order dynamics (1). Given $x_{\mathrm{d}} \in \mathcal{F}$, the control protocol (9)-(11) guarantees the collision-free navigation to $x_{\mathrm{d}}$ from almost all initial conditions $\left(x\left(t_{0}\right), v\left(t_{0}\right), \hat{m}\left(t_{0}\right), \hat{\alpha}\left(t_{0}\right)\right) \in \mathcal{F} \times \mathbb{R}^{n+1} \times \mathbb{R}_{\geq 0}$, given a sufficiently small $\tau$ and that $k_{\phi}>\frac{\alpha}{2}$. Moreover, all closed loop signals remain bounded, $\forall t \geq t_{0}$.

Proof. See the proof of Theorem 1 in Verginis and Dimos (2020).

Remark 1. Note that, unlike the related works in feedbackbased robot navigation, the proposed algorithm guarantees almost global safe convergence while compensating for unknown dynamic terms ( $f$ and $m$ in this case). Moreover, in contrast to tuning schemes (e.g., Dimarogonas et al., 2006; Koditschek \& Rimon, 1990; Loizou, 2011; Vlantis et al., 2018), we do not require large control gains in order to establish the correctness of the propose scheme.

Remark 2. The condition $k_{\phi}>\frac{\alpha}{2}$ of Theorem 1 is only sufficient and not necessary, as will be shown in the simulation results. Moreover, in case the robot gets stuck in a local minimum, one could apply an exciting input perpendicular to $x-x_{\mathrm{d}}$ (see Vrohidis et al., 2018), freeing it thus from that configuration. Nevertheless, the set of initial conditions that drive the robot to such configurations has zero measure and hence the probability of starting in it is zero. ${ }^{2}$

\footnotetext{
2 The exciting input could be applied at the initial condition, if it can be identified that it will lead to a local minimum.
} 


\subsection{Dynamic disturbance addition}

Except for the already considered dynamic uncertainties, we can add to the right-hand side of (1) an unknown disturbance vector $d(x, v, t)$, i.e.,

$\dot{x}=v$

$m \dot{v}+f(x, v)+m g+d(x, v, t)=u$,

subject to a uniform boundedness condition $\|d(x, v, t)\| \leq \bar{d}$, $\forall x, v, t \in \mathbb{R}^{2 n} \times \mathbb{R}_{\geq 0}$. In this case, by slightly modifying the control scheme, we still guarantee collision avoidance with the workspace obstacles and boundary. In addition, we achieve uniform ultimate boundedness of the error signals as well as the gradient of $\phi$, as the analysis in this section shows.

The control scheme of the previous section is appropriately enhanced to incorporate the $\sigma$-modification (Lavretsky \& Wise, 2013), a common technique in adaptive control. More specifically, the adaptation laws (11) are modified according to

$\begin{aligned} \dot{\hat{m}} & :=-k_{m} e_{v}^{\top}\left(\dot{v}_{\mathrm{d}}+g\right)-\sigma_{m} \hat{m} \\ \dot{\hat{\alpha}} & :=k_{\alpha}\left\|e_{v}\right\|^{2}-\sigma_{\alpha} \hat{\alpha},\end{aligned}$

where $\sigma_{m}, \sigma_{\alpha}$ are positive gain constants. More specifically, the addition of $\sigma_{m}, \sigma_{\alpha}$ guarantees the uniform boundedness of $\hat{m}, \hat{\alpha}$. By exploiting the boundedness of $d(\cdot)$ and the fact that $\lim _{d_{j} \rightarrow 0} \beta\left(d_{j}\right)=\infty$, one can easily show that collisions are provably avoided. Although the goal configuration is not proven to be almost globally stabilized, intuition suggests that if $d(\cdot)$ does not behave adversarially, the agent will converge close to it. More details can be found in Section 4.1 of Verginis and Dimos (2020).

\section{Extension to star worlds}

In this section, we discuss how the proposed control scheme can be extended to generalized sphere worlds, and in particular star worlds, being inspired by the methodology of Rimon and Koditschek (1992). That work however, like others related to workspace transformations (Loizou, 2014; Vlantis et al., 2018), considers simplified dynamics without taking into account unknown terms, which is the focus of this section. Although we focus on star-worlds, the analysis holds for any diffeomorphic transformation that exhibits the desired properties (e.g. Vlantis et al., 2018). Star worlds are diffeomorphic to sphere worlds sets of the form $\mathcal{T}:=\overline{\mathcal{W}} \backslash \bigcup_{j \in \mathcal{J}} \bar{O}_{\mathcal{T}_{j}}$, where $\overline{\mathcal{W}}$ is a workspace of the form (4) and $\bar{O}_{\mathcal{T}_{j}}$ are $M$ disjoint star-shaped obstacles (indexed by $\mathcal{J}=\{1, \ldots, M\})$. The latter are sets characterized by the property that all rays emanating from a center point cross their boundary only once (Rimon \& Koditschek, 1992). One can design a diffeomorphic mapping $H: \mathcal{T} \rightarrow \mathcal{F}$, where $\mathcal{F}$ is a sphere world of the type (5). More specifically, $H$ maps the boundary of $\mathcal{T}$ to the boundary of $\mathcal{F}$. Construction of such a mapping is beyond the scope of the paper and we refer the interested reader to the related literature (Rimon \& Koditschek, 1991, 1992).

The control scheme of the previous section is modified now to account for the transformation $H$ as follows: The desired robot velocity is set to $v_{\mathrm{d}}: \mathcal{T} \rightarrow \mathbb{R}^{n}$, with

$v_{\mathrm{d}}(x):=-J_{H}(x)^{-1} \nabla_{H(x)} \phi(H(x))$,

where $J_{H}(x):=\frac{\partial H(x)}{\partial x}$ is the nonsingular Jacobian matrix of $H$. Next, by letting $e_{v}^{\partial x}:=v-v_{\mathrm{d}}$, the control law is designed as $u: \mathcal{T} \times \mathbb{R}^{n+2} \rightarrow \mathbb{R}^{n}$, with

$$
\begin{aligned}
u:=u(x, v, \hat{m}, \hat{\alpha}):= & -k_{\phi} J_{h}(x)^{\top} \nabla_{H(x)} \phi(H(x))+ \\
& \hat{m}\left(\dot{v}_{\mathrm{d}}+g\right)-\left(k_{v}+\frac{3}{2} \hat{\alpha}\right) e_{v},
\end{aligned}
$$

where $\hat{m}$ and $\hat{\alpha}$ evolve according to the respective expressions in (11). The next theorem gives the main result of this section.
Theorem 2. Consider a robot operating in $\mathcal{W}$, subject to the uncertain 2nd-order dynamics (1). Given $x_{\mathrm{d}} \in \mathcal{T}$, the control protocol (11)-(13) guarantees the collision-free navigation to $x_{\mathrm{d}}$ from almost all initial conditions $\left(x\left(t_{0}\right), v\left(t_{0}\right), \hat{m}\left(t_{0}\right), \hat{\alpha}\left(t_{0}\right)\right) \in \mathcal{T} \times \mathbb{R}^{n_{1}} \times \mathbb{R}_{\geq 0}$, given a sufficiently small $\tau$ and that $k_{\phi}>\frac{\alpha}{2}$. Moreover, all closed loop signals remain bounded, $\forall t \geq t_{0}$.

Proof. See the proof of Theorem 2 in Verginis and Dimos (2020).

Remark 3. The proposed schemes can also be extended to unknown environments, where the amount and location of the obstacles is unknown a priori, and these are sensed locally online. In particular, by having a large enough sensing neighborhood, each obstacle $j \in \mathcal{J}$ can be sensed when $d_{j}=\tau$, and hence the respective term can be smoothly incorporated in $\nabla_{x} \phi(x)$, in view of the properties of $\beta$ (a similar idea is discussed in Section 5 of Paternain et al., 2017). It should be noted, however, that the local sensory information and respective hardware must allow for the accurate estimation of the centers and radii (or the implicit function in case of star-worlds) of the obstacles.

\section{Extension to multi-robot systems}

This section is devoted to extending the results of Section 4 to multi-robot systems. Consider, therefore, $N \in \mathbb{N}$ spherical robots operating in a workspace $\mathcal{W}$ of the form (3), characterized by their position vectors $x_{i} \in \mathbb{R}^{n}$, as well as their radii $r_{i}>0$, $i \in \mathcal{N}:=\{1, \ldots, N\}$, and obeying the second-order uncertain dynamics (1), i.e.,

$\dot{x}_{i}=v_{i}$

$m_{i} \dot{v}_{i}+f_{i}\left(x_{i}, v_{i}\right)+m_{i} g=u_{i}$,

with the unknown $f_{i}(\cdot)$ satisfying $\left\|f_{i}\left(x_{i}, v_{i}\right)\right\| \leq \alpha_{i}\left\|v_{i}\right\|$, for unknown positive constants $\alpha_{i}, \forall i \in \mathcal{N}$. We also denote $x:=$ $\left[x_{1}^{\top}, \ldots, x_{N}^{\top}\right]^{\top}, v:=\left[v_{1}^{\top}, \ldots, v_{N}^{\top}\right]^{\top} \in \mathbb{R}^{N n}$. Each robot's destination is $x_{\mathrm{d}_{i}}, i \in \mathcal{N}$.

The proposed multi-robot scheme is based on a prioritized leader-follower coordination. Prioritization in multi-agent systems for navigation-type objectives has been employed in Guo, Tumova, and Dimarogonas (2016) and Roussos and Kyriakopoulos (2013), where KRNF gain tuning-type methodologies are developed. The proposed framework, however, is substantially different from these works; Guo et al. (2016) do not take into account inter-agent collisions, and use prioritization for the sequential navigation and task satisfaction subject to connectivity constraints, while Roussos and Kyriakopoulos (2013) use prioritization for directional collision-avoidance. In our proposed prioritized leader-follower methodology, the leader robot, by appropriately choosing the offset $\tau$, "sees" the other robots as static obstacles and hence the overall scheme reduces to the one of Section 4 . This is accomplished by differentiating the free spaces of the leader and the followers. Moreover, the aforementioned works Guo et al. (2016) and Roussos and Kyriakopoulos (2013) consider simplified first-order dynamics and cannot be easily extended to the uncertain dynamics-case considered here. In fact, we note that, according to our best knowledge, there does not exist a control framework that provably guarantees decentralized safe multi-robot navigation in workspaces with obstacles and subject to uncertain 2nd-order dynamics.

The workspace is assumed to satisfy Assumption 2 and we further impose the following extra conditions:

Assumption 3. The workspace $\mathcal{W}$, obstacles $\mathcal{O}_{j}, j \in \mathcal{J}$, and destinations $x_{\mathrm{d}_{i}}, i \in \mathcal{N}$, satisfy:

$\left\|c_{j}-x_{\mathrm{d}_{i}}\right\|>r_{o_{j}}+r_{i}+2 r_{M}+\varepsilon, \forall i, j \in \mathcal{N} \times \mathcal{J}$ 
$\left\|x_{\mathrm{d}_{i}}-x_{\mathrm{d}_{j}}\right\|>r_{i}+r_{j}+2 r_{M}+2 \varepsilon, \forall i, j \in \mathcal{N}, i \neq j$

$r_{\mathcal{W}}-\left\|x_{\mathrm{d}_{i}}\right\|>r_{i}+2 r_{M}+\varepsilon, \forall i \in \mathcal{N}$

whereas the initial positions satisfy:

$\left\|c_{j}-x_{i}\left(t_{0}\right)\right\|>r_{o_{j}}+r_{i}+2 r_{M}, \forall i, j \in \mathcal{N} \times \mathcal{J}$

$r_{\mathcal{W}}-\left\|x_{i}\left(t_{0}\right)\right\|>r_{i}+2 r_{M}, \forall i \in \mathcal{N}$

$\left\|x_{\mathrm{d}_{i}}-x_{j}\left(t_{0}\right)\right\|>r_{i}+r_{j}+2 r_{M}+\varepsilon, \forall i, j \in \mathcal{N}, i \neq j$,

for an arbitrarily small positive constant $\varepsilon, \forall i \in \mathcal{N}, j \in \mathcal{J}$, where $r_{M}:=\max _{i \in \mathcal{N}}\left\{r_{i}\right\}$.

Loosely speaking, the aforementioned assumption states that the pairwise distances among obstacles, workspace boundary, initial conditions and final destinations are large enough so that one robot can always navigate between them. Since the convergence of the agents to their destinations is asymptotic, we incorporate the threshold $\varepsilon$, which is the desired proximity we want to achieve to the destination, as will be clarified in the sequel. Intuitively, since we cannot achieve $x_{i}=x_{\mathrm{d}_{i}}$ in finite time, the high-priority agents will stop once $\left\|x_{i}-x_{\mathrm{d}_{i}}\right\|=\varepsilon$, which is included in the aforementioned conditions to guarantee the feasibility of the collision-free navigation for the lower-priority agents. Similarly to the single-agent case, we can find a positive constant $\bar{r}$ such that (6) hold as well as

$$
\begin{aligned}
& \left\|c_{j}-x_{i}\left(t_{0}\right)\right\|>r_{o_{j}}+r_{i}+2 r_{M}+2 \bar{r}, \forall i, j \in \mathcal{N} \times \mathcal{J} \\
& r_{\mathcal{W}}-\left\|x_{i}\left(t_{0}\right)\right\|>r_{i}+2 r_{M}+2 \bar{r}, \forall i \in \mathcal{N} \\
& \left\|c_{j}-x_{\mathrm{d}_{i}}\right\|>r_{o_{j}}+r_{i}+2 r_{M}+\varepsilon+2 \bar{r}, \forall i, j \in \mathcal{N} \times \mathcal{J} \\
& \left\|x_{\mathrm{d}_{i}}-x_{\mathrm{d}_{j}}\right\|>r_{i}+r_{j}+2 r_{M}+2 \varepsilon+2 \bar{r}, \forall i, j \in \mathcal{N}, i \neq j \\
& \left\|x_{\mathrm{d}_{i}}-x_{j}\left(t_{0}\right)\right\|>r_{i}+r_{j}+2 r_{M}+\varepsilon+2 \bar{r}, \forall i, j \in \mathcal{N}, i \neq j \\
& r_{\mathcal{W}}-\left\|x_{\mathrm{d}_{i}}\right\|>r_{i}+2 r_{M}+\varepsilon+2 \bar{r}, \forall i \in \mathcal{N}
\end{aligned}
$$

We consider that the agents have a limited sensing range, defined by a radius $\varsigma_{i}>0, i \in \mathcal{N}$, and we assume that each agent $i$ can sense the state of its neighbors:

Assumption 4. Each agent $i \in \mathcal{N}$ has a limited sensing radius $\varsigma_{i}$, satisfying $\zeta_{i}>\sqrt{\min \left(\bar{r}^{2}, \bar{r}_{\mathrm{d}}\right)}+r_{i}+r_{j}+2 r_{M}+2 \bar{r}$, with $\bar{r}_{\mathrm{d}}$ as defined in (7), and has access to $x_{i}-x_{j}, v_{i}-v_{j}, \forall j \in\left\{j \in \mathcal{N}:\left\|x_{i}-x_{j}\right\| \leq \varsigma_{i}\right\}$.

Moreover, we consider that the destinations, $x_{\mathrm{d}_{i}}, i \in \mathcal{N}$, as well as the radii, $r_{i}$, are transmitted off-line to all the agents. ${ }^{3}$ Consider now a prioritization of the agents, possibly based on some desired metric (e.g., distance to their destinations), which can be performed off-line and transmitted to all the agents. Our proposed scheme is based on the following algorithm. The agent with the highest priority is designated as the leader of the multiagent system, indexed by $i_{\mathcal{L}}$, whereas the rest of the agents are considered as the followers, defined by the set $\mathcal{N}_{\mathcal{F}}:=\mathcal{N} \backslash\left\{i_{\mathcal{L}}\right\}$. The followers and leader employ a control protocol that has the same structure as the one of Section 4. The key difference here lies in the definition of the free space for followers and leaders. Let $q=\left[q_{1}^{\top}, \ldots, q_{N}^{\top}\right]^{\top} \in \mathbb{R}^{N n}$. We define first the sets

$\overline{\mathcal{W}}_{i_{\mathcal{L}}}:=\left\{q \in \mathbb{R}^{N n}:\left\|q_{i_{\mathcal{L}}}\right\|<r_{\mathcal{W}}-r_{i_{\mathcal{L}}}\right\}$,

$\overline{\mathcal{O}}_{i_{\mathcal{L}}, j}:=\left\{q \in \overline{\mathcal{W}}_{i_{\mathcal{L}}}:\left\|q_{i}-c_{j}\right\| \leq r_{o_{j}}+r_{i}\right\}, \forall j \in \mathcal{J}$

$\mathcal{C}_{i_{\mathcal{L}}}:=\left\{q \in \overline{\mathcal{W}}_{i_{\mathcal{L}}}:\left\|q_{i_{\mathcal{L}}}-q_{j}\right\| \leq r_{i_{\mathcal{L}}}+r_{j}, \forall j \in \mathcal{N} \backslash\left\{i_{\mathcal{L}}\right\}\right\}$,

which correspond to the leader agent, as well as the follower sets

$\overline{\mathcal{W}}_{i}:=\left\{q \in \mathbb{R}^{N n}:\left\|q_{i}\right\|<r_{\mathcal{W}}-r_{i}-2 r_{M}-2 \bar{r}\right\}$

$\overline{\mathcal{O}}_{i, j}:=\left\{q \in \overline{\mathcal{W}}_{i}:\left\|q_{i}-c_{j}\right\| \leq r_{o_{j}}+r_{i}+2 r_{M}+2 \bar{r}\right\}, \forall j \in \mathcal{J}$

3 This implies that the agents can compute $r_{M}$ offline.

$$
\begin{aligned}
\mathcal{C}_{i}:=\left\{q \in \overline{\mathcal{W}}_{i}:\left\|q_{i}-q_{i_{\mathcal{L}}}\right\| \leq r_{i}+r_{i_{\mathcal{L}}},\right. \\
\\
\quad\left\|q_{i}-q_{j}\right\| \leq r_{i}+r_{j}+2 r_{M}+2 \bar{r}, \forall j \in \mathcal{N} \backslash\left\{i_{\mathcal{L}}, i\right\}, \\
\left.\quad\left\|q_{i}-x_{\mathrm{d}_{j}}\right\| \leq r_{i}+r_{j}+2 r_{M}+2 \bar{r}+\varepsilon, \forall j \in \mathcal{N}_{i}\right\},
\end{aligned}
$$

$\forall i \in \mathcal{N}_{\mathcal{F}}$, where $\mathcal{N}_{i}$ denotes the set of agents with higher priority than agent $i$. The free space for the agents is defined then as $\mathcal{F}_{i}:=\overline{\mathcal{W}}_{i} \backslash\left\{\left(\bigcup_{j \in \mathcal{J}} \overline{\mathcal{O}}_{i, j}\right) \cup \mathcal{C}_{i}\right\}, \forall i \in \mathcal{N}$. It can be verified that, in view of (15), the sets $\mathcal{F}_{i}$ are nonempty and $x\left(t_{0}\right) \in \mathcal{F}:=\bigcap_{i \in \mathcal{N}} \mathcal{F}_{i}$. The main difference lies in the fact that the follower agents aim to keep a larger distance from each other, the obstacles, and the workspace boundary than the leader agent, and in particular, a distance enhanced by $2 r_{M}+2 \bar{r}$. In that way, the leader agent will be able to choose an appropriate constant $\tau$ (as in the singleagent case of Section 4) so that it is influenced at each time instant only by one of the obstacles/followers, and will be also able to navigate among the obstacles/followers. Note that the followers are required to stay away also from the destinations of the higher priority agents, since a potential local minimum in such configurations can prevent the leader agent from reaching its goal. We provide next the mathematical details of the aforementioned reasoning.

Consider the leader distances $d_{i_{\mathcal{L}}, o_{k}}, d_{i_{\mathcal{L}}, j}, d_{i_{\mathcal{L}}, o_{0}}: \mathcal{F}_{i_{\mathcal{L}}} \rightarrow \mathbb{R}_{\geq 0}$ as

$d_{i_{\mathcal{L}}, o_{k}}(x):=\left\|x_{i_{\mathcal{L}}}-c_{k}\right\|^{2}-\left(r_{i_{\mathcal{L}}}+r_{o_{k}}\right)^{2}, \forall k \in \mathcal{J}$

$d_{i_{\mathcal{L}}, j}(x):=\left\|x_{i_{\mathcal{L}}}-x_{j}\right\|^{2}-\left(r_{i_{\mathcal{L}}}+r_{j}\right)^{2}, \forall j \in \mathcal{N}_{\mathcal{F}}$

$d_{i_{\mathcal{L}}, o_{0}}(x):=\left(r_{\mathcal{W}}+r_{i_{\mathcal{L}}}\right)^{2}-\left\|x_{i_{\mathcal{L}}}\right\|^{2}$

and the follower distances $d_{i, o_{k}}, d_{i, i_{\mathcal{L}}}, d_{i, j}, d_{i, \mathrm{~d}_{j}} d_{i, o_{0}}: \mathcal{F}_{i} \rightarrow \mathbb{R}_{\geq 0}$ as

$d_{i, o_{k}}(x):=\left\|x_{i}-c_{k}\right\|^{2}-\left(r_{i}+r_{o_{k}}+2 r_{M}+2 \bar{r}\right)^{2}, \forall k \in \mathcal{J}$

$d_{i, i_{\mathcal{L}}}(x):=\left\|x_{i}-x_{i_{\mathcal{L}}}\right\|^{2}-\left(r_{i}+r_{i_{\mathcal{L}}}\right)^{2}=d_{i_{\mathcal{L}}, i}(x)$

$d_{i, j}(x):=\left\|x_{i}-x_{j}\right\|^{2}-\left(r_{i}+r_{j}+2 r_{M}+2 \bar{r}\right)^{2}, \forall j \in \mathcal{N}_{\mathcal{F}} \backslash\{i\}$

$d_{i, \mathrm{~d}_{j}}(x):=\left\|x_{i}-x_{\mathrm{d}_{j}}\right\|^{2}-\left(r_{i}+r_{j}+2 r_{M}+2 \bar{r}+\varepsilon\right)^{2}, \forall j \in \mathcal{N}_{i}$

$d_{i, o_{0}}(x):=\left(r_{\mathcal{W}}-r_{i}-2 r_{M}-2 \bar{r}\right)^{2}-\left\|x_{i}\right\|^{2}$,

$\forall i \in \mathcal{N}_{\mathcal{F}}$. Note that $d_{i, j}(x)=d_{j, i}(x), \forall i, j \in \mathcal{N}$, with $i \neq j$ and also that $x \in \mathcal{F}$ is equivalent to all the aforementioned distances being positive.

Let now functions $\beta, \beta_{i}, i \in \mathcal{N}$, that satisfy the properties of Definition 1 , as well as the respective constants $\tau$, $\tau_{i}$, such that $\beta^{\prime}(z)=\beta^{\prime \prime}(z)=0, \forall z \geq \tau, \beta_{i}^{\prime}(z)=\beta_{i}^{\prime \prime}(z)=0, \forall z \geq \tau_{i}, i \in \mathcal{N}$. The 2nd-order navigation functions for the agents are now defined as $\phi_{i}: \mathcal{F}_{i} \rightarrow \mathbb{R}_{\geq 0}$, with

$$
\begin{array}{rlrl}
\phi_{i}(x) & :=k_{1_{i}}\left\|x_{i}-x_{\mathrm{d}_{i}}\right\|^{2}+k_{2_{i}}\left(b_{1_{i}}(x)+b_{2_{i}}(x)+k_{f_{i}} b_{3_{i}}(x)\right) \\
b_{1_{i}}(x) & :=\sum_{j \in \overline{\mathcal{J}}} \beta_{i}\left(d_{i, o_{j}}(x)\right), & b_{2_{i}}(x) & :=\sum_{j \in \mathcal{N} \backslash\{i\}} \beta\left(d_{i, j}(x)\right) \\
b_{3_{i}}(x) & :=\sum_{j \in \mathcal{N}_{i}} \beta_{i}\left(d_{i, \mathrm{~d}_{j}}(x)\right),
\end{array}
$$

$\forall i \in \mathcal{N}$, and $k_{f_{i} \mathcal{L}}=0, k_{f_{i}}=1, \forall i \in \mathcal{N}_{\mathcal{F}}$. Note that the robotic agents can choose independently their $\tau_{i}, i \in \mathcal{N}$, that concerns the collision avoidance with the obstacles and the workspace boundary. The pair-wise inter-agent distances, however, are required to be the same and hence the same $\beta$ (and hence $\tau$ ) is chosen (see the terms $b_{2_{i}}(x)$ in $\phi_{i}(x)$ ), which can, nevertheless, be done off-line. To achieve convergence of the leader to its destination, we choose $\tau$ and $\tau_{i_{\mathcal{L}}}$ as in Section 4, i.e., $\tau, \tau_{i_{\mathcal{L}}} \in\left(0, \min \left\{\bar{r}^{2}, \bar{r}_{\mathrm{d}}\right\}\right)$. In view of Assumption 4, it can be proved that $d_{i, j}(x)<\tau$ implies $\left\|x_{i}-x_{j}\right\| \leq \varsigma_{i}, \forall i, j \in \mathcal{N}, i \neq j$.

The control protocol follows the same structure as the singleagent case presented in Section 4. In particular, we define the 

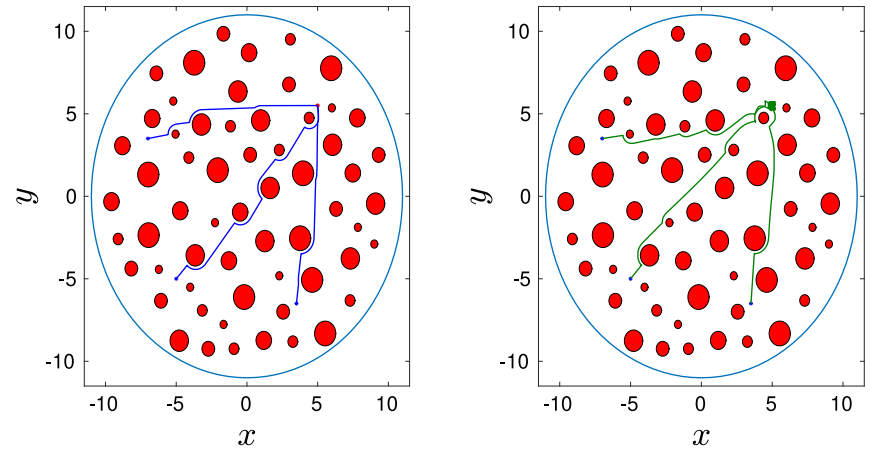

Fig. 1. The resulting trajectories $x(t), t \in[0,100] \mathrm{s}$, from the initial points $-(5,5),(-7,3.5)$, and $(3.5,-7)$ to the destination $(5,5)$. Left: without any disturbances. Right: with bounded disturbance $d(x, v, t)$.
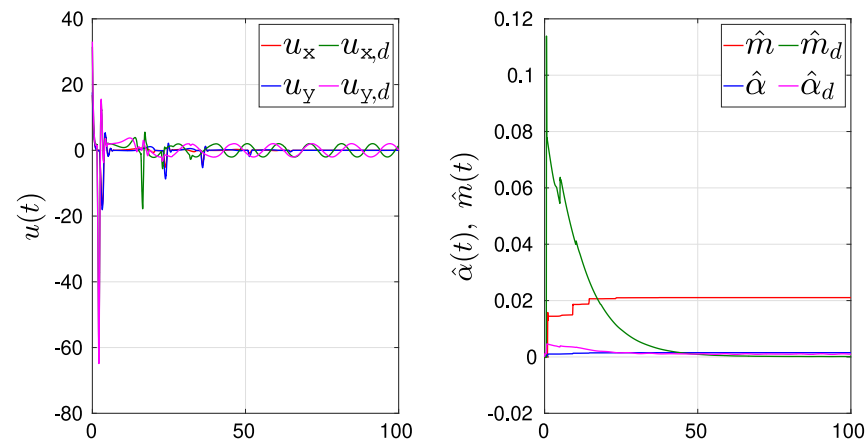

Fig. 2. The input $u(t)=\left(u_{1}(t), u_{2}(t)\right)$ (left), and adaptation signals $\hat{\alpha}(t), \hat{m}(t)$ (right) for the 2D trajectory from $-(5,5)$ to $(5,5)$ of Fig. 1. The subscript $d$ corresponds to the model where a bounded disturbance vector $d(x, v, t)$ was included.

reference velocities as $v_{\mathrm{d}_{i}}: \mathcal{F}_{i} \rightarrow \mathbb{R}^{n}$, with

$v_{\mathrm{d}_{i}}(x):=-\nabla_{x_{i}} \widetilde{\phi}_{i}(x)$,

where $\widetilde{\phi}_{i}: \mathcal{F}_{i} \rightarrow \mathbb{R}_{\geq 0}$ is the slightly modified function:

$\widetilde{\phi}_{i}(x):=k_{1_{i}}\left\|x_{i}-x_{\mathrm{d}_{i}}\right\|^{2}+k_{2_{i}}\left(b_{1_{i}}(x)+2 b_{2_{i}}(x)+k_{f_{i}} b_{3_{i}}(x)\right)$

The need for modification of $\phi_{i}$ to $\widetilde{\phi}_{i}$ stems from the differentiation of the terms $b_{2_{i}}$. The control law is designed as $u_{i}:=$ $u_{i}\left(x, v, \hat{m}_{i}, \hat{\alpha}_{i}\right): \mathcal{F}_{i} \times \mathbb{R}^{N n+2} \rightarrow \mathbb{R}^{n}$, with

$u_{i}:=-k_{\phi_{i}} \nabla_{x_{i}} \widetilde{\phi}_{i}(x)+\hat{m}_{i}\left(\dot{v}_{\mathrm{d}_{i}}+g\right)-\left(k_{v_{i}}+\frac{3}{2} \hat{\alpha}_{i}\right) e_{v_{i}}$

$\forall i \in \mathcal{N} ; k_{\phi_{i}}, k_{v_{i}}$ are positive constants, $e_{v_{i}}$ are the velocity errors $e_{v_{i}}:=v_{i}-v_{\mathrm{d}_{i}}$, and $\hat{m}_{i}, \hat{\alpha}_{i}$ denote the estimates of $m_{i}$ and $\alpha_{i}$, respectively, by agent $i$, evolving according to (11). We further denote $\hat{m}:=\left[\hat{m}_{1}, \ldots, \hat{m}_{N}\right]^{\top}, \hat{\alpha}:=\left[\hat{\alpha}_{1}, \ldots, \hat{\alpha}_{N}\right]^{\top} \in \mathbb{R}^{N}$. The following theorem considers the convergence of a leader to its destination.

Theorem 3. Consider $N$ robots operating in $\mathcal{W}$, subject to the uncertain 2nd-order dynamics (14), and a leader $i_{\mathcal{L}}$. Under Assumption 1-4, the control protocol (16), (17), (11) guarantees collision avoidance between the agents and the agents and obstacles/workspace boundary as well as convergence of $x_{i_{\mathcal{L}}}$ to $x_{\mathrm{d}_{i_{\mathcal{L}}}}$ from almost all initial conditions $\left(x\left(t_{0}\right), v\left(t_{0}\right), \hat{m}\left(t_{0}\right), \hat{\alpha}\left(t_{0}\right)\right) \in \mathcal{F} \times$ $\mathbb{R}^{N(n+1)} \times \mathbb{R}_{\geq 0}^{N}$, given sufficiently small $\tau, \tau_{i_{\mathcal{L}}}$, and that $k_{\phi_{i}}>\frac{\alpha_{i}}{2}$, $i \in \mathcal{N}$. Moreover, all closed loop signals remain bounded, $\forall t \geq t_{0}$.

Proof. See the proof of Theorem 3 in Verginis and Dimos (2020).
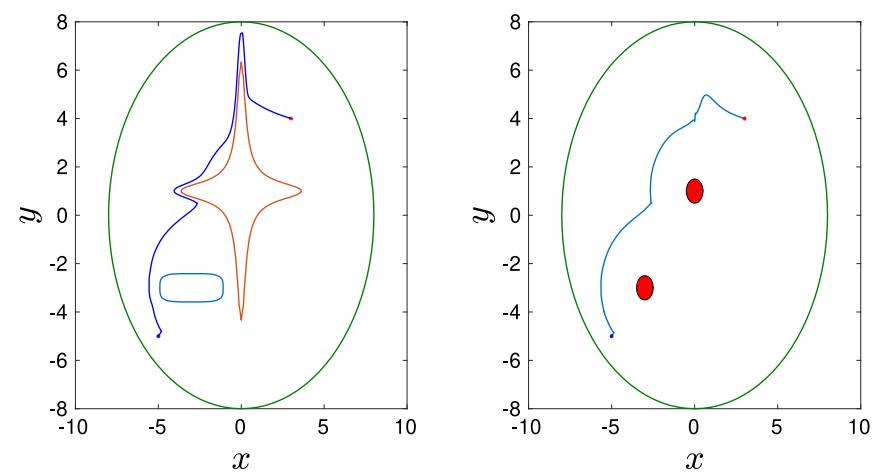

Fig. 3. The resulting trajectory $x(t), t \in[0,500] \mathrm{s}$, from the initial points $-(5,5)$ to the destination $(3,4)$, in the $2 \mathrm{D}$ star world workspace (left) and the transformed sphere world (right).

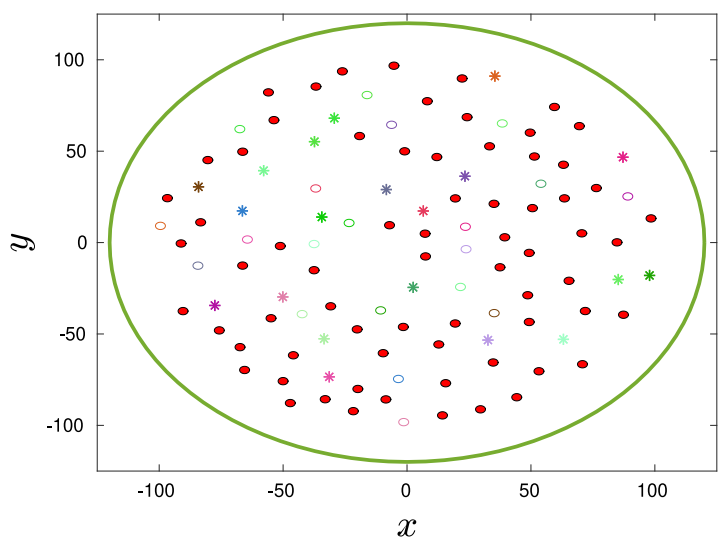

Fig. 4. The initial configurations of the multi-agent scenario. The obstacles are depicted as filled red disks whereas the agents as circles. The destinations are shown with asterisk. (For interpretation of the references to color in this figure legend, the reader is referred to the web version of this article.)

When the current leader $i_{\mathcal{L}}$ reaches $\varepsilon$-close to its goal, at a time instant $t_{i_{\mathcal{L}}},{ }^{4}$ it broadcasts this information to the other agents, switches off its control and remains immobilized, considered hence as a static obstacle with center $c_{M+1}:=x_{i_{\mathcal{L}}}\left(t_{i_{\mathcal{L}}}\right)$ and radius $r_{M+1}$ by the rest of the team. Note that $\left\|c_{M+1}-x_{\mathrm{d}_{i_{\mathcal{L}}}}\right\| \leq \varepsilon$ and hence, in view of (15), $\left\|c_{j}-c_{M+1}\right\|>r_{o_{j}}+r_{i_{\mathcal{L}}}+2 r_{M}+2 \bar{r}$, $\forall j \in \mathcal{J}$, and $r_{\mathcal{W}}-\left\|c_{M+1}\right\|>r_{i_{\mathcal{L}}}+2 r_{M}+2 \bar{r}$, satisfying the obstacle spacing properties (6). The next agent $i_{\mathcal{L}}^{\prime} \in \tilde{\mathcal{N}}:=\mathcal{N} \backslash\left\{i_{\mathcal{L}}\right\}$ in priority is then assigned as a leader for navigation, and we redefine the sets

$\widetilde{\overline{\mathcal{O}}}_{i^{\prime}}, j:=\left\{q \in \overline{\mathcal{W}}_{i_{\mathcal{L}}^{\prime}}:\left\|q_{i}-c_{j}\right\| \leq r_{o_{j}}+r_{i}\right\}, \forall j \in \widetilde{\mathcal{J}}$

$\widetilde{\mathcal{C}}_{i_{\mathcal{L}}^{\prime}}:=\left\{q \in \overline{\mathcal{W}}_{i_{\mathcal{L}}^{\prime}}:\left\|q_{i_{\mathcal{L}}^{\prime}}-q_{j}\right\| \leq r_{i_{\mathcal{L}}^{\prime}}+r_{j}, \forall j \in \tilde{\mathcal{N}} \backslash\left\{i_{\mathcal{L}}^{\prime}\right\}\right\}$,

$\widetilde{\overline{\mathcal{O}}}_{i, j}:=\left\{q \in \overline{\mathcal{W}}_{i}:\left\|q_{i}-c_{j}\right\| \leq r_{o_{j}}+r_{i}+2 r_{M}+2 \bar{r}\right\}, \forall j \in \tilde{\mathcal{J}}$

$\widetilde{\mathcal{C}_{i}}:=\left\{q \in \overline{\mathcal{W}}_{i}:\left\|q_{i}-q_{i^{\prime}}\right\| \leq r_{i}+r_{i_{\mathcal{C}}^{\prime}}\right.$,

$$
\begin{aligned}
& \left\|q_{i}-q_{j}\right\| \leq r_{i}+r_{j}+2 r_{M}+2 \bar{r}, \forall j \in \tilde{\mathcal{N}} \backslash\left\{i_{\mathcal{L}}^{\prime}, i\right\}, \\
& \left.\left\|q_{i}-x_{\mathrm{d}_{j}}\right\| \leq r_{i}+r_{j}+2 r_{M}+2 \bar{r}+\varepsilon, \forall j \in \widetilde{\mathcal{N}}_{i}\right\},
\end{aligned}
$$

$\forall i \in \tilde{\mathcal{N}} \backslash\left\{i_{\mathcal{L}}^{\prime}\right\}$, where $\tilde{\mathcal{N}}_{i}:=\mathcal{N}_{i} \backslash\left\{i_{\mathcal{L}}\right\}$, and $\tilde{\mathcal{J}}:=\mathcal{J} \cup\{M+1\}$, to account for the new obstacle $M+1$. The new free space is $\widetilde{\mathcal{F}}_{i}:=\overline{\mathcal{W}}_{i} \backslash\left\{\left(\bigcup_{j \in \widetilde{\mathcal{J}}} \widetilde{\overline{\mathcal{O}}}_{i, j}\right) \cup \widetilde{\mathcal{C}}_{i}\right\}, \forall i \in \widetilde{\mathcal{N}}$ and one can conclude that $x_{i^{\prime}}\left(t_{i_{\mathcal{L}}}\right) \in \widetilde{\mathcal{F}}_{i^{\prime}}, x_{i}\left(t_{i_{\mathcal{L}}}\right) \in \widetilde{\mathcal{F}}_{i} \forall i \in \tilde{\mathcal{N}} \backslash\left\{i_{\mathcal{L}}^{\prime}\right\}$. Therefore, the application of Theorem 3 with $t_{i_{\mathcal{L}}}$ as $t_{0}$ and agent $i_{\mathcal{L}}^{\prime}$ as leader guarantees

\footnotetext{
4 Note that the proven asymptotic stability of Theorem 3 guarantees that this
} will occur in finite time. 

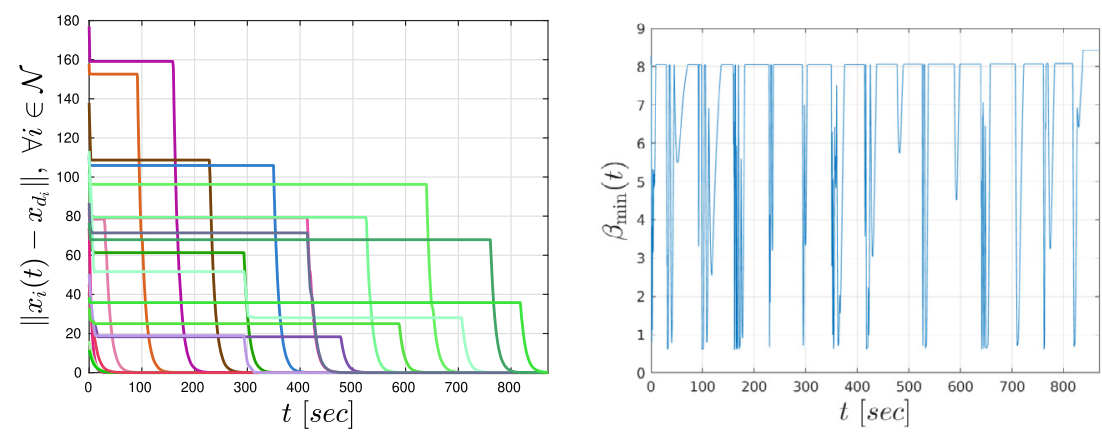

Fig. 5. The resulting signals $\left\|x_{i}(t)-x_{\mathrm{d}_{i}}\right\|, \forall i \in \mathcal{N}$ (left) and the signal $\beta_{\min }(t)$ (right).

its navigation $\varepsilon$-close to $x_{\mathrm{d}_{i^{\prime}}}$. Applying iteratively the aforementioned reasoning, we guarantee the successful navigation of all the agents to their destinations. Finally, we note that due to prioritization-based leader-follower architecture, the total time to reach the goals increases with the size of the multi-agent team.

\section{Simulation results}

This section verifies the theoretical findings of Sections 4-6 via computer simulations. We consider first a $2 \mathrm{D}$ workspace on the horizontal plane with $r_{\mathcal{W}}=11$, populated with $M=60$ randomly placed obstacles, whose radius, enlarged by the robot radius, is randomly chosen in $\bar{r}_{o_{j}} \in[0.25,0.75], \forall j \in \mathcal{J}$. The mass, is chosen as $m=1$, and $f(x, v)=\frac{\alpha}{16} \sin \left(0.5\left(x_{1}+x_{2}\right)\right) F(v) v$, with $F(v)=\operatorname{diag}\left\{\left[\exp \left(-\operatorname{sgn}\left(v_{i}\right) v_{i}\right)+1\right]_{i \in\{1,2\}}\right\}$, and $\alpha=10$, where we denote $\left(x_{1}, x_{2}\right)=x,\left(v_{1}, v_{2}\right)=v$. Note that $f()$ is highly nonlinear, motivated by the friction model of De Wit, Olsson, Astrom, and Lischinsky (1995). We choose the goal position as $x_{\mathrm{d}}=(5,5)$, which the robot aims to converge to from 3 different initial positions, namely $x(0)=-(5,5),(-7,3.5)$, and $(3.5,-7)$. The parameter $\bar{r}$ is chosen as $\bar{r}=0.5$. We choose a variation of (8) for $\beta$ with $\tau=\bar{r}^{2}$. The control gains are chosen as $k_{1}=0.04$, $k_{2}=5, k_{v}=20, k_{\phi}=1$, and $k_{m}=k_{\alpha}=0.01$. The results for $t \in[0,100] \mathrm{s}$ are depicted in Figs. 1, 2; 1 (left) shows that the robot navigates to its destination without any collisions, and 2 depicts the input and adaptation signals $u(t), \hat{\alpha}(t), \hat{m}(t)$ for the trajectory starting from $(-5,5)$. In addition, note that the fact that $\alpha>2$ does not affect the performance of the proposed control protocol and hence we can verify that the condition $k_{\phi}>\frac{\alpha}{2}$ is only sufficient and not necessary. Moreover, in order to verify the results of Remark 2, we add a bounded time-varying disturbance vector $d(x, v, t)=d(t):=2\left[\sin \left(0.5 t+\frac{p i}{3}\right), \cos \left(0.4 t-\frac{\pi}{4}\right)\right]^{\top} \in \mathbb{R}^{2}$ and we choose the extra control gains as $\sigma_{m}=\sigma_{\alpha}=0.1$. The results are depicted in Fig. 1 (right), which shows the safe navigation of the agent to a set close to $x_{\mathrm{d}}$, and Fig. 2, which shows the input and adaptation signals for the trajectory starting from $(-5,5)$.

Next, we illustrate the performance of the control protocol of Section 5 in a 2D star-world. We consider a workspace with $r_{\mathcal{W}}=$ 8 , which contains 2 star-shaped obstacles, centered at $(-3,-3)$ and $(0,1)$, respectively. The mass $m$ and function $f(x, v)$ are given as before, with $\alpha=1$. In order to transform the workspace to a sphere world, we employ the transformation proposed in Rimon and Koditschek (1992). In the transformed sphere world, we choose $\bar{r}=4$ and $\bar{r}_{o_{j}}=0.5$, whereas the function $\beta$ is chosen as in the sphere-world case. The initial and goal position are selected as $x(0)=(-5,-5)$ and $x_{\mathrm{d}}=(3,4)$, respectively, and the control gains as $k_{1}=0.04, k_{2}=.2, k_{v}=20, k_{\phi}=1$, and $k_{m}=k_{\alpha}=0.01$. The robot trajectory is depicted in Fig. 3, for $t \in[0,500] \mathrm{s}$, both in the original star and in the transformed sphere world.
Finally, we use the control scheme of Section 6 in a multiagent scenario. We consider 20 agents in a 2D workspace of $r_{\mathcal{W}}=120$, populated with 70 obstacles. The agents and obstacles are randomly initialized to satisfy the conditions of the free space of Section 6, as shown in Fig. 4. The radius of the agents and the obstacles is chosen as $r_{i}=r_{o_{j}}=2, \forall i \in \mathcal{N}, j \in \mathcal{J}$, and the sensing radius of the agents is taken as $\zeta_{i}=20, \forall i \in \mathcal{N}$. The functions $\beta, \beta_{i}$ are chosen as before, and we also choose $\bar{r}=4, \varepsilon=0.1$. The results are depicted in Fig. 5 for 870 $\mathrm{s}$, which shows the convergence of the distance errors $\| x_{i}(t)-$ $x_{\mathrm{d}-i} \|$ to zero, $\forall i \in \mathcal{N}$ as well as the minimum of the distances $\left\|x_{i}(t)-x_{j}(t)\right\|-2 r, \forall i, j \in \mathcal{N}, i \neq j$, and $\left\|x_{i}(t)-c_{j}\right\|-2 r$, $\forall i \in \mathcal{N}, j \in \mathcal{J}$, defined as $\beta_{\min }(t):=\min \left\{\min _{i, j \in \mathcal{N}, i \neq j}\left\{\| x_{i}(t)-\right.\right.$ $\left.\left.x_{j}(t) \|-2 r\right\}, \min _{(i, j) \in \mathcal{N} \times \mathcal{J}}\left\{\left\|x_{i}(t)-c_{j}\right\|-2 r\right\}\right\}$, which stays strictly positive, $\forall t \in[0,870]$, implying that collisions are avoided. A video illustrating the multi-agent navigation can be found in https://vimeo.com/393443782.

\section{Conclusion and future work}

This paper considers the robot navigation in an obstaclecluttered environment subject to uncertain 2nd-order dynamics. A novel navigation function is proposed and combined with adaptation laws that compensate for the uncertain dynamics. The results are extended to star worlds as well as multi-agent cases. Future directions will aim at relaxing the assumptions for the latter.

\section{References}

Alonso-Mora, Javier, Breitenmoser, Andreas, Rufli, Martin, Siegwart, Roland, \& Beardsley, Paul (2012). Image and animation display with multiple mobile robots. International Journal of Robotics Research, 31(6), 753-773.

Arslan, Omur, Guralnik, Dan P., \& Koditschek, Daniel E. (2016). Coordinated robot navigation via hierarchical clustering. IEEE Transactions on Robotics, 32(2), 352-371.

Arslan, Omur, \& Koditschek, Daniel E. (2016). Exact robot navigation using power diagrams. In International Conference on Robotics and Automation (ICRA) (pp. 1-8). IEEE.

Arslan, Omur, \& Koditschek, Daniel E. (2017). Smooth extensions of feedback motion planners via reference governors. In International Conference on Robotics and Automation (ICRA) (pp. 4414-4421). IEEE.

Berkane, Soulaimane, Bisoffi, Andrea, \& Dimarogonas, Dimos V. (2019). A hybrid controller for obstacle avoidance in an n-dimensional euclidean space. In European Control Conference (pp. 764-769). IEEE.

Cheah, Chien Chern, Hou, Saing Paul, \& Slotine, Jean Jacques E. (2009). Region-based shape control for a swarm of robots. Automatica, 45(10), 2406-2411.

De Wit, C Canudas, Olsson, Hans, Astrom, Karl Johan, \& Lischinsky, Pablo (1995). A new model for control of systems with friction. Transactions on Automatic Control, 40(3), 419-425.

Dimarogonas, Dimos V, Loizou, Savvas G, Kyriakopoulos, Kostas J, \& Zavlanos, Michael M (2006). A feedback stabilization and collision avoidance scheme for multiple independent non-point agents. Automatica, 42(2), 229-243. 
Filippidis, Ioannis, \& Kyriakopoulos, Kostas J. (2011). Adjustable navigation functions for unknown sphere worlds. In Conference on Decision and Control and European Control Conference (pp. 4276-4281). IEEE.

Filotheou, Alexandros, Nikou, Alexandros, \& Dimarogonas, Dimos V. (2018). Decentralized control of uncertain multi-agent systems with connectivity maintenance and collision avoidance. In European Control Conference (pp. 8-13). IEEE.

Grushkovskaya, V., \& Zuyev, A. (2018). Obstacle avoidance problem for second degree nonholonomic systems. In Conference on Decision and Control (pp. 1500-1505). IEEE.

Guo, Meng, Tumova, Jana, \& Dimarogonas, Dimos V. (2016). Communicationfree multi-agent control under local temporal tasks and relative-distance constraints. IEEE Transactions on Automatic Control, 61(12), 3948-3962.

Huber, Lukas, Billard, Aude, \& Slotine, Jean-Jacques (2019). Avoidance of convex and concave obstacles with convergence ensured through contraction. IEEE Robotics and Automation Letters, 4(2), 1462-1469.

Koditschek, Daniel E. (1991). The control of natural motion in mechanical systems. Journal of Dynamic Systems Measurement and Control, 113(4), 547-551.

Koditschek, Daniel E., \& Rimon, Elon (1990). Robot navigation functions on manifolds with boundary. Advances in Applied Mathematics, 11(4), 412-442.

LaValle, Steven M. (2006). Planning algorithms. Cambridge university press.

Lavretsky, Eugene, \& Wise, Kevin A. (2013). Robust adaptive control. In Robust and adaptive control (pp. 317-353). Springer.

Lionis, Grigoris, Papageorgiou, Xanthi, \& Kyriakopoulos, Kostas J. (2007). Locally computable navigation functions for sphere worlds. In International Conference on Robotics and Automation (ICRA) (pp. 1998-2003). IEEE.

Loizou, Savvas G. (2011). Closed form navigation functions based on harmonic potentials. In Conference on Decision and Control and European Control Conference (pp. 6361-6366). IEEE.

Loizou, Savvas G. (2014). The multi-agent navigation transformation: Tuning-free multi-robot navigation. Robotics: Science and Systems, 6, 1516-1523.

Loizou, Savvas G. (2017). The navigation transformation. IEEE Transactions on Robotics, 33(6), 1516-1523.

Lumelsky, Vladimir J. (2005). Sensing, intelligence, motion: how robots and humans move in an unstructured world. John Wiley \& Sons.

Makkar, C., Dixon, W. E., Sawyer, W. G., \& Hu, G. (2005). A new continuously differentiable friction model for control systems design. In IEEE/ASME International Conference on Advanced Intelligent Mechatronics (pp. 600-605). IEEE.

Mendes Filho, José M., Lucet, Eric, \& Filliat, David (2017). Real-time distributed receding horizon motion planning and control for mobile multi-robot dynamic systems. In International Conference on Robotics and Automation (ICRA) (pp. 657-663). IEEE.

Montenbruck, Jan Maximilian, Bürger, Mathias, \& Allgöwer, Frank (2015). Navigation and obstacle avoidance via backstepping for mechanical systems with drift in the closed loop. In American Control Conference (pp. 625-630). IEEE.

Morgan, Daniel, Subramanian, Giri P., Chung, Soon-Jo, \& Hadaegh, Fred Y. (2016). Swarm assignment and trajectory optimization using variable-swarm, distributed auction assignment and sequential convex programming. The International Journal of Robotics Research, 35(10), 1261-1285.

Panagou, Dimitra (2017). A distributed feedback motion planning protocol for multiple unicycle agents of different classes. Transactions on Automatic Control, 62(3), 1178-1193.

Paternain, Santiago, Koditschek, Daniel E., \& Ribeiro, Alejandro (2017). Navigation functions for convex potentials in a space with convex obstacles. IEEE Transactions on Automatic Control, 63(9), 2944-2959.

Rimon, Elon, \& Koditschek, Daniel E. (1991). The construction of analytic diffeomorphisms for exact robot navigation on star worlds. Transactions of the American Mathematical Society, 327(1), 71-116.

Rimon, Elon, \& Koditschek, Daniel E. (1992). Exact robot navigation using artificial potential functions. Transactions on Robotics and Automation, 8(5), 501-518.

Roussos, Giannis, \& Kyriakopoulos, Kostas J. (2013). Decentralized and prioritized navigation and collision avoidance for multiple mobile robots. In Distributed Autonomous Robotic Systems (pp. 189-202). Springer.

Stavridis, Sotiris, Papageorgiou, Dimitrios, \& Doulgeri, Zoe (2017). Dynamical system based robotic motion generation with obstacle avoidance. IEEE Robotics and Automation Letters, 2(2), 712-718.
Szulczyński, Paweł, Pazderski, Dariusz, \& Kozłowski, Krzysztof (2011). Real-time obstacle avoidance using harmonic potential functions. Journal of Automation Mobile Robotics and Intelligent Systems, 5, 59-66.

Tanner, Herbert G., \& Kumar, Amit (2005). Towards decentralization of multirobot navigation functions. In International Conference on Robotics and Automation (ICRA) (pp. 4132-4137). IEEE.

Van Den Berg, Jur, Snape, Jamie, Guy, Stephen J., \& Manocha, Dinesh (2011). Reciprocal collision avoidance with acceleration-velocity obstacles. In International Conference on Robotics and Automation (ICRA). IEEE.

Verginis, Christos K., \& Dimarogonas, Dimos V. (2017). Robust decentralized abstractions for multiple mobile manipulators. In Conference on Decision and Control (pp. 2222-2227). IEEE.

Verginis, K. Christos, \& Dimarogonas, V. Dimos (2019). Closed-form barrier functions for multi-agent ellipsoidal systems with uncertain Lagrangian dynamics. IEEE Control Systems Letters (L-CSS).

Verginis, C. K., \& Dimos, V. D. (2020). Adaptive robot navigation with collision avoidance subject to 2nd-order uncertain dynamics. ArXiv link: https://arxiv. org/pdf/2005.12599.pdf.

Verginis, Christos K., Nikou, Alexandros, \& Dimarogonas, Dimos V. (2018). Communication-based decentralized cooperative object transportation using nonlinear model predictive control. In European Control Conference (pp. 733-738). IEEE.

Verginis, Christos K, Xu, Ziwei, \& Dimarogonas, Dimos V (2017). Decentralized motion planning with collision avoidance for a team of uavs under high level goals. In International Conference on Robotics and Automation (ICRA) (pp. 781-787). IEEE.

Vlantis, Panagiotis, Vrohidis, Constantinos, Bechlioulis, Charalampos P, \& Kyriakopoulos, Kostas J (2018). Robot navigation in complex workspaces using harmonic maps. In International Conference on Robotics and Automation (ICRA) (pp. 1726-1731). IEEE.

Vrohidis, Constantinos, Vlantis, Panagiotis, Bechlioulis, Charalampos P, \& Kyriakopoulos, Kostas J (2018). Prescribed time scale robot navigation. IEEE Robotics and Automation Letters, 3(2), 1191-1198.

Wang, Li, Ames, Aaron D., \& Egerstedt, Magnus (2017). Safety barrier certificates for collisions-free multirobot systems. IEEE Transactions on Robotics, 33(3), 661-674.

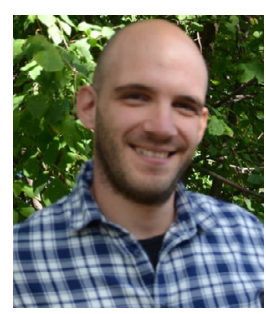

Christos K. Verginis was born in Athens, Greece in 1989. He received the Diploma in electrical and computer engineering and M.Sc. degree in Automatic Control Systems and Robotics from the National Technical University of Athens (NTUA), Greece, in 2013 and 2015, respectively. He received the Ph.D. in electrical engineering from the Royal Institute of Technology (KTH), Stockholm, Sweden, in 2020. His research interests include multiagent systems, formal verification, and control of unknown systems.

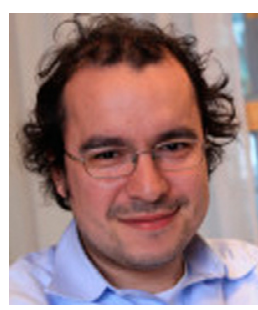

Dimos V. Dimarogonas was born in Athens, Greece, in 1978. He received the Diploma in Electrical and Computer Engineering in 2001 and the Ph.D. in Mechanical Engineering in 2007, both from National Technical University of Athens (NTUA), Greece. Between May 2007 and February 2009, he was a Postdoctoral Researcher at the Department of Automatic Control, School of Electrical Engineering and Computer Science, Royal Institute of Technology (KTH), Stockholm, Sweden. Between February 2009 and March 2010, he was a Postdoctoral Associate at the Laboratory for Information and Decision Systems (LIDS) at the Massachusetts Institute of Technology (MIT), Boston, MA, USA. He is currently Professor at the Division of Decision and Control, KTH Royal Institute of Technology, Stockholm, Sweden. His current research interests include Multi-Agent Systems, Hybrid Systems and Control, Robot Navigation and Networked Control. He serves in the Editorial Board of Automatica, the IEEE Transactions on Control of Network Systems and the Unmanned Systems journal and is a Senior member of IEEE and the Technical Chamber of Greece. 